\title{
Multilinguales
}

Multilinguales

$10 \mid 2018$

Langues et médias dans les milieux plurilingues

\section{Quand la musique (re)gagne la rue : corps et discriminations en contexte urbain au}

\section{Cameroun}

hen the music (re)invades the street: body and discriminations in Cameroon

urban context

\author{
Jean-Benoît Tsofack
}

\section{(2) OpenEdition}

Journals

Édition électronique

URL : http://journals.openedition.org/multilinguales/3648

DOI : $10.4000 /$ multilinguales.3648

ISSN : 2335-1853

Éditeur

Université Abderrahmane Mira - Bejaia

Référence électronique

Jean-Benoît Tsofack,

"Quand la musique (re)gagne la rue : corps et discriminations en contexte urbain auCameroun », Multilinguales [En ligne], 10 | 2018, mis en ligne le 31 décembre 2018, consulté le 10 décembre 2020.

URL : http://journals.openedition.org/multilinguales/3648; DOI : https://doi.org/10.4000/

multilinguales.3648

Ce document a été généré automatiquement le 10 décembre 2020.

\section{cc)}

Multilinguales est mise à disposition selon les termes de la Licence Creative Commons Attribution -

Pas d'Utilisation Commerciale - Pas de Modification 4.0 International 


\section{Quand la musique (re)gagne la rue : corps et discriminations en contexte urbain au}

\section{Cameroun}

hen the music (re)invades the street: body and discriminations in Cameroon

urban context

Jean-Benoît Tsofack

1 La musique urbaine camerounaise contemporaine est progressivement devenue l'un des espaces des discours sociaux ou des arts populaires en général où la discrimination positivée du corps a connu un véritable délitement ces dix dernières années. Elle a migré des préoccupations sociales réelles ${ }^{1}$ vers une mise en scène ou en évidence du sexe et de la pornographie à travers des paroles infâmes noyées dans le tintamarre des sonorités et des percussions qui l'accompagnent. La musique a tout simplement (re)gagné la rue ${ }^{2}$ ou tout au moins la rue a envahi la musique comme la plupart des langues et des discours qui les charrient en contexte urbain notamment. Elle a bousculé les normes (esthétiques, sociales et voire artistiques) avec une évolution thématique au niveau du «bas ventre » qui prône l'obscène et une désacralisation de la nudité et de l'intimité qui étaient jusque-là le socle des valeurs traditionnelles ou culturelles.

2 Comment donc comprendre cette rupture esthétique soudaine ? Comment comprendre et expliquer ces pratiques discursives discriminantes révélatrices des tensions et des fragmentations sociales dans l'espace public? Comment comprendre enfin le discours pornographique comme une nouvelle forme de reconfiguration de l'espace social au Cameroun? Telles sont les pistes que j'entends explorer dans cette contribution pour rester dans la thématique des neuvièmes Journées Internationales de Sociolinguistique 
Urbaine (JISU) tenues à l'Université de Rennes 2 en mai-juin 2018 ${ }^{3}$. L'objectif est ici d'explorer le discours artistique comme étant une nouvelle forme de (ré)appropriation et de réinvention de l'espace social public pouvant nécessiter une régulation sociale et politique dans le but de consolider le « vivre ensemble».

Les observables qui structurent cette réflexion se situent dans le prolongement d'une série de recherches menées conjointement par mes étudiants (Ndoungmo (2017) ${ }^{4}$, Tilong $(2017)^{5}$,) et moi-même (Sotso et Tsofack, en cours ${ }^{6}$ ) depuis 2013 sur la musique urbaine camerounaise. Je m'appuie sur un corpus non sollicité d'extraits de paroles de musiques transcrites par moi-même chez quatre artistes musiciens contemporains couvrant la période allant de 2013 à 2017 : Maalhox Le Vibeur (Tu montes tu descends (2016)) ; Franco (Coller la petite (2015)) ; Petit Pays (Papa est sorti (2013)) et Petit Malo (Les Go kamer (2016).

4 L'approche relève majoritairement de l'Analyse du discours dans son versant sociologique comme pratique sociale par excellence et repose sur le principe théorique des normes sociales et des discriminations en sociolinguistique urbaine. Je commencerai dans un premier temps par présenter un bref aperçu de la musique camerounaise en général et urbaine en particulier, ensuite je visiterai les différents aspects des discriminations du corps observées dans la parole musicale et enfin j'envisagerai les pistes d'intervention possibles dans l'optique d'une régulation sociale et politique.

\section{Normes sociales et discriminations: des perspectives en sociolinguistique urbaine}

5 La sociolinguistique urbaine, telle qu'on l'envisage (Bulot et Veschambre, 2007) s'est donnée pour objectif entre autres de contribuer à redonner vigueur au questionnement fondateur de la sociolinguistique : quelles réponses la discipline peut-elle apporter face à l'exclusion des minorités sociales? Il est question ici d'explorer les rapports complexes entre la production des normes (sociales, artistiques, etc.) et les dynamiques discriminatoires, et plus particulièrement de voir en quoi les espaces urbains (la ville) de référence se constituent en normes non seulement socio-spatiales mais encore des vecteurs / facteurs des normes langagières, et, partant, des processus discriminatoires, des dynamiques comparées de frontérisation (Bulot, $2013: 7$ ).

6 On lui assigne ainsi, pour reprendre les premières théorisations relatives à la militance sociale (Bulot, 2001; Bulot et Beauvois, 2004), l'ambitieuse mission de réfléchir sur les différentes façons de lutter contre les discriminations toutes les fois que les pratiques langagières sont impliquées (Bulot, 2007: 31). Il s'agit là d'une volonté du sociolinguiste, et donc du chercheur à la dénonciation de l'exclusion des minorités sociales pour ce que ce dernier processus produit bien entendu de la souffrance individuelle mais aussi de délitement du lien social, du lien communautaire. On pourrait ainsi postuler une sociolinguistique de l'intervention (Bulot, 2009a et 2009b) où les chercheurs sont à la fois des acteurs qui ont légitimité à intervenir sur des situations urbaines constatées, celles où les faits de ségrégations sociales se comprennent comme des phénomènes construits socialement comme étant nécessaires voire indispensables. 


\section{La musique dans la rue : refaire l'espace public ?}

7 De manière générale, on peut noter dans l'histoire de la musique camerounaise trois grands moments ${ }^{7}$ : les années 50 et 60 avec les musiques folkloriques (le Bikutsi et l'Assiko) ayant un ancrage fort dans la vie sociale et les réalités culturelles locales, puis les années 70-80 avec le Makossa, genre populaire le plus répandu ayant fait le succès des grands noms au Cameroun et à l'étranger. A partir des années 90 , une nouvelle ère musicale se met en place à la faveur de l'ouverture au monde extérieur, des contestations politiques et sociales ayant contraint à l'ouverture démocratique, mais surtout à l'invasion des nouvelles technologies de l'information et de la communication. Il s'agit d'une musique-mosaïque ayant une liberté de ton qui capitalise les ressentiments socio-politiques divers et ouvre à la contestation des styles anciens, de l'ordre politique ambiant et des injustices sociales naissantes. On parlera même de la subversion des codes et des normes artistiques, esthétiques ou éthiques prévalents à la faveur de l'émergence et de la promotion d'une culture dite urbaine dont naîtront en conséquence les tendances musicales dites aussi urbaines.

\subsection{Musique urbaine, Mboa urban music, Hip hop Kamer...}

8 L'expression musique urbaine a été utilisée pour la première fois dans l'industrie du disque aux Etats-Unis à travers le terme Urban music (Meyran, 2014) dans les années 80. Les musiques urbaines sont à comprendre comme un mélange de plusieurs genres musicaux populaires et contemporains qui touchent un large public et sont pensées comme des musiques métissées et de fusion prenant place dans une "bulle urbaine » (la cité, la rue, les périphéries ou encore les quartiers, etc.) (TNK, 2016). Elles sont les musiques de rupture et en rupture qui promeuvent un réagencement original de divers codes culturels, une déconstruction d'oppositions esthétiques et une subversion des normes qui structurent classiquement la musique occidentale. C'est bien dans ce contexte de renouvellement, de rupture et de reconfiguration des codes et normes esthétiques qu'il faut situer la musique urbaine camerounaise encore appelée Mboa urban music (Atome, 2017) ou musiques Génération Mboa (Atome, 2016). Le terme luimême est formé à partir de la racine Mboa qui marque ici la camerounité et le parcours qui a construit ces tendances musicales, et du caractérisant urban music pour revenir à l'appellation de base de la musique urbaine. De manière simplifiée, l'on peut utiliser indistinctement les termes Mboa music ou Musiques Mboa pour désigner ou se référer à la tendance musicale dite urbaine au Cameroun.

9 La musique urbaine camerounaise a ses caractéristiques qui lui sont propres aux points de vue technique ou esthétique qui permettent de la distinguer d'autres tendances musicales existantes. Ces caractéristiques sont notamment : l'instrumental à cadence hip hop ou contenant une ligne de sonorités hiphop, les lyrics et les textes exploitant les codes linguistiques (Camfranglais, Français et Anglais camerounais, Pidgin-english, langues nationales) et la réalité environnementale ou socio-culturelle camerounais, la fusion aux rythmes Camerounais (Benskin-makossa-bikutsi...), le flow et le ton Camerounais, et surtout la personnalité du chanteur qui doit être d'origine ou de nationalité camerounaise. La langue utilisée étant ici majoritairement le camfranglais qui est la langue de la liberté par excellence, on peut comprendre que son secret réside « dans le verbe » (Diallo, 2017) et son discours, dans la rue ! 


\subsection{La rue dans la musique et le corps dans tous ses états !} se réclame, fait une part belle à l'indécence et à la décadence qui caractérisent le climat social de l'heure. Il s'agit non seulement de repenser la parole publique dans un espace de liberté, mais aussi de revisiter et promouvoir le corps comme un système de valeurs, dans un espace artistique où la popularité rime avec l'obscénité. Nouveau repère pour la jeunesse urbaine, l'espace social connaît une telle déconstruction des frontières et une extension des limites de la décence qu'il suffit désormais de chanter des «banalités » pour se hisser au sommet de la popularité (Ambombo, 2016). On est donc ainsi loin de l'époque où la musique populaire camerounaise rimait avec éthique et moralité, la période des musiques «bien travaillées " étant révolue, c'est plutôt la musique banale faite de chansons et chorégraphies obscènes qui captivent toutes les attentions et suscitent toutes les curiosités

11 De manière générale, l'espace public au Cameroun est loin d'être vu ou perçu comme un espace homogène en tous points de vue (géographique, linguistique, culturel, social...) et plus particulièrement en ce qui concerne l'approche genre ou les représentations du corps et de la féminité. On peut ainsi observer dans la parole musicale urbaine plusieurs formes d'injustices ou de maltraitances verbales du corps: une première tendance est celle liée à la pratique sexuelle elle-même présentée comme une activité coercitive, un acte non négocié et réalisé dans la brutalité. Dans ce cas, et selon la plupart des musiciens urbains, l'acte sexuel ne se présente pas comme une activité mutuellement consentie par les parties prenantes, mais comme un acte subi sous fond de violence :

Les parents ne sont pas là, profitez, amusez-vous, dansez, sautez, mangez, croquez, cognez (Petit Pays ; 2013)

Peu importe la taille ou la couleur, moi j'entre seulement dedans (Maalhox, 2016)

Collez, collez, collez, colle la petite (Franco, 2015)

De toutes les façons même si l'acte sexuel du fait des commodités sociales ou discursives est désigné sous la forme métaphorique dans la musique urbaine, il est à noter qu'il représente dans l'ensemble une activité coercitive qui fait le lit de la violence, celle d'un corps sur un autre qui s'apparente à un viol ou un crime organisé. On n'a qu'à noter le sémantisme des verbes d'action utilisés à chaque fois comme cogner, croquer, coller, mettre dedans, etc. qui dénote de la brutalité.

Tu montes tu descends, aujourd'hui je vais seulement mettre ça dedans (Maalhox, 2016)

Récupère la petite, embrouille la petite et maintenant colle la petite (Franco, 2015)

On est loin des conventions sociales en la matière qui veulent que dans ce domaine le rapport de forces soit équitable et la décision mutuellement consentie. La musique entend ainsi instaurer un nouvel ordre social fondé sur la transgression verbale des normes, le sabotage des valeurs sociales :

Choisis ta petite, mange-la avec appétit (Franco, 2015)

Moi je sors mon déodorant je parfume bien ma chose et tu connais la suite (Maalhox, 2016) 

appartenance ou son assignation à un espace géographique initialement valorisé ou dévalorisé. Les femmes de certaines régions du Cameroun par exemple sont socialement étiquetées pour certaines pratiques corporelles :

La fille bassa ${ }^{8}$, elle adore le way ${ }^{9}$ (Petit Malo, 2016)

On dit que les filles Maka ${ }^{10}$ adorent le sassayé ${ }^{11}$ (Petit Malo, 2016) la musique entend ici renseigner sur la vision de la femme camerounaise par l'opinion publique (Ndoungmo (2017: 27), avec des clichés discriminants ou stigmatisants :

La fille bamiléké ${ }^{12}$ excès de fécondité accouche les enfants et ça attire la pauvreté (Petit Malo, 2016)

La fille Bamoun adore la saleté. Quand elles vont se laver, elles lavent seulement les pieds (Petit Malo, 2016)

Les filles Nanga ${ }^{13}$ adorent le farotage ${ }^{14}$ (Petit Malo, 2016) affiche, aux dires des musiciens, une légèreté des mœurs profondément ancrée dans un déterminisme ontologique mis en exergue dans les origines socio-culturelles. On comprend donc que la femme n'est pas seulement dégradée ou ravalée, voire chosifiée, mais son corps est désacralisé par le discours musical urbain.

ne troisième tendance est celle liée à la discrimination dans la nomination/ désignation, bref, de la mise en mots du corps ou des parties intimes du corps qui consacre l'impolitesse comme une catégorie pertinente. Dans ce paradigme, il convient de distinguer deux situations : la première est celle liée au vocabulaire ou aux formes de désignation du sexe de la femme qui sont essentiellement dépréciatives ou réifiantes :

(13) Mon totem c'est le rat c'est pourquoi je cherche toujours le trou (Maalhox, 2016)

(14) Mbom je mets seulement dedans après je vérifie si c'est un chat mort ou un chat vivant (Maalhox, 2016)

(15) Quand c'est une affaire de piment massa ne mets jamais ta bouche (Maalhox, 2016)

(16) Je parfume bien les bêtises, je continue (Maalhox, 2016)

18 La plupart des expressions métaphoriques utilisées ici pour désigner le sexe de la femme relèvent du langage familier avec un registre ordurier, qu'il s'agisse de bêtise, de piment ou du trou. Le sexe de la femme est donc un simple organe banalisé voire désacralisé à côté du sexe de l'homme à qui on attribue des caractéristiques virilisantes ou offensives. Il faut noter ici qu'à côté du l'organe sexuel féminin, certaines parties saillantes du corps comme les seins et les fesses subissent également une souscatégorisation discriminative axiologique gradante ou dégradante :

(17) Il y a tous les genres de lasses (Franco, 2015)

(18) Il y a tout genre de lolos (Franco, 2015)

19 La deuxième situation dans cette catégorie elle celle liée aux rapports sociaux ou aux relations interpersonnelles et notamment aux formes nominales d'adresse marquées par l'invective et le mépris. Si de manière générale on peut considérer la violence comme toute atteinte à l'intégrité d'une personne selon plusieurs sphères, notamment psychologique, économique, sexuelle et autres avec ou sans l'assentiment de la victime (Ndoungmo, 2017: 45), on peut bien comprendre qu'il s'agit d'une force déréglée qui porte atteinte à l'intégrité physique pour mettre en cause dans un but de domination 
ou de destruction de l'individu. C'est d'abord sous forme de mépris que le corps est envisagé :

(19) Tu dis que le dedans est sale mais tu es toujours dedans (Maahlox, 2016)

(20) Tu dis que le dedans sent mais tu es toujours dedans (Maahlox, 2016)

On peut donc comprendre ces formes de violence symbolique manifestée dans l'insulte et le mépris comme étant la "forme ultime du conflit symbolique» (Stewart, 2007 : 174) qui marque une rupture avec le decorum discursif imposé par l'élite, et le rejet de son mode de raisonnement. Le discours musical est ici le reflet de l'environnement social global du Cameroun en proie à la décadence :

(21) Je t'assure ici tout le monde est fou (Franco, 2015)

21 Cette " folie » sociale est donc remarquable dans tous les aspects de la vie publique, et l'on peut comprendre que la musique n'est que la partie visible, l'arbre qui cache la forêt de ce délabrement moral qui appelle et nécessite une régulation politique allant dans le sens de l'assainissement.

\section{Dénoncer, intervenir, s'impliquer?}

Ce titre qui reprend partiellement celui d'une publication sous la direction de Thierry Bulot (2009a) rejoint les préoccupations de la sociolinguistique urbaine qui pose la nécessité d'une intervention (sociale ou politique) dans le cadre d'une sociolinguistique dite "prioritaire». Le discours musical urbain au Cameroun en effet, est manifestement un discours de la pourriture du corps physique certes, mais beaucoup plus du corps social et/ou moral qui exige et nécessite une intervention où des acteurs divers doivent s'impliquer pour réguler et améliorer non seulement les inégalités et les injustices de toutes sortes, mais aussi le climat social dans le cadre de la promotion du vivre ensemble. A ce niveau trois axes d'intervention sont possibles et demandent à être renforcées :

Le premier axe est celui de la sensibilisation sur les inégalités et les discriminations par les chercheurs de tous bords à travers leurs résultats de la recherche, mais aussi leur implication possible dans l'aménagement de l'espace public comme un capital social et symbolique. Ndoungmo (2017: 56 et svtes) par exemple avait déjà pu évaluer la réception de la musique urbaine à travers les réactions du public sur internet qui lui ont permis de mesurer l'impact de ces musiques sur les consommateurs. Elle a pu montrer par exemple que, malgré le fait que le titre Coller la petite de Franko par exemple ait été frappé d'interdiction, il a cependant connu un succès fulgurant du point de vue du nombre de personnes ayant regardé, écouté ou « liké » son clip vidéo sur You Tube, en capitalisant entre 2015 et 2017 plus de 47.822.042 "vues », 209000 "j'aime " contre 15000 «j'aime pas " seulement. De même, Tu montes tu descends de Maahlox a pu engranger 3.332.242 de "vues", 9.518 de "j'aime" contre 1.606 de «j'aime pas». Mais cette réception positive a aussi été inversement proportionnelle à la vague de condamnation qui s'est soulevée dans et en dehors des réseaux sociaux dans la société camerounaise en général.

Le deuxième axe est celui de la dénonciation et de la condamnation, notamment par les acteurs de la société civile que sont les internautes comme dans le cas précédent, mais aussi de certains musiciens de la tendance dite classique. Dans cette direction, certains artistes musiciens condamnent l'irresponsabilité et l'amateurisme professionnelles 
chez les musiciens de la nouvelle génération. Pour Blaise Essame, ingénieur de son (cité in Ambombo, 2015), la musique populaire camerounaise connait « une chute brutale avec des influences extérieures. Les artistes ne veulent plus travailler. Ils adorent la facilité, surtout avec la prépondérance des ordinateurs ». Ils ont oublié, disait Ama Pierrot (cité in Ambombo (op.cit.), qu'une chanson est comme une rédaction, elle demande « une introduction, un développement et une conclusion pour que le résultat soit impeccable »

Le troisième axe est celui de l'action qu'on peut envisager sous un double aspect social et politique. Sur le plan social, on peut envisager une censure des mœurs notamment chez les gardiens des valeurs morales que sont les hommes d'église. Dans une correspondance adressée aux artistes, Alexis Godonou (2014) pasteur de son état, adresse une mise en garde solennelle à tous ceux-là qui, par des actes qu'ils posent "contribuent à l'annihilation de la morale et de l'éthique dans notre société ». Il conclut sa correspondance en invitant à une intervention politique, notamment les ministres, les élus locaux, les responsables de la société des droits d'auteurs et autres chefs traditionnels qui devraient «se mobiliser et dire non à cette croisade de destruction de notre jeunesse ». A côté de la censure des mœurs, on peut envisager une censure professionnelle pouvant déboucher sur des sanctions diverses. La première mesure se trouverait du côté du peuple et donc des consommateurs qui doivent "sanctionner le mauvais grain de la culture comme il a l'habitude de le faire dans les urnes lors des différentes élections ". Ange Ebogo (cité in Ambombo, op. cit.) lui aussi pose la nécessité d'une régulation institutionnelle à travers "une commission de censure ».

Sur le plan politique on pourrait restaurer la censure administrative qui avait longuement prospéré dans le champ médiatique. On peut, dans cette optique se référer par exemple à l'interdiction par les autorités administratives, notamment le Prefet de la Mifi (Bafoussam) à l'Ouest-Cameroun, de la chanson coller la petite de Franko, entre autres.

Pour ne pas conclure cette réflexion, il est utile de reprendre et de mentionner que la sociolinguistique urbaine est une sociolinguistique dite "de crise» (Bulot, 2008) qui s'attache à repérer les tensions sociales, les faits de ségrégation et les pratiques discriminatoires, tels qu'ils se manifestent, mais aussi se construisent dans et par les discours au sein d'un espace social très largement modifié et perturbé par l'urbanisation. A cet égard, l'espace social défini par la musique urbaine camerounaise est majoritairement un espace de construction et de valorisation des inégalités, voire des injustices corporelles qui donnent à voir à une crise sociale nourrie par une crise politique.

28 Le discours musical est perverti par un vocabulaire obscène qui est la matérialisation même des tensions et des fragmentations d'une société en crise. Face à une telle situation, une action urgente est envisageable dans une double direction sociale et politique afin d'assainir et réguler un espace public camerounais où le vivre ensemble est sérieusement mis en mal ces derniers temps par des fractures identitaires diverses dont la récente « crise anglophone » est la forme de cristallisation la plus patente. 


\section{BIBLIOGRAPHIE}

АМВОМво М., " Cameroun-musique : quand popularité rime avec obscénité ", article mis en ligne en 2016. Disponible sur https://www.237online.com/article-08487-cameroun--musiquequand-popularite-rime-avec-obscenite.html. Consulté le 16.05.2018.

ATOME, «Comment définir la musique urbaine au Cameroun? », article mis en ligne en 2016. Disponible sur http://voilamoi.over-blog.com/2016/02/comment-definir-musique-urbaine-aucameroun.html. Consulté le 16.05.2018.

ATOME , « Aux origines de la musique urbaine camerounaise. Du « Hip hop kamer » à la « Mboa urban music » ", article mis en ligne en 2017. Disponible sur http://www.voila-moi.com/auxorigines-de-la-musique-urbaine-camerounaise-hip-hop-kamer-mboa-urban-music/. Consulté le 16.05.2018.

BULOT, T., « Ségrégation et urbanisation linguistique : l'altérité urbaine définie ou « l'étranger est une personne » ", Diverscité langues (en ligne), vol. VI Québec, Téléuniversité, 2001, 22 p. Disponible sur [http://www.teluq.uquebec.ca/diverscite/entree.htm]. Consulté le 16.5.2018.

BULOT, T., «Culture urbaine et diversité sociolinguistique : une identité en mouvement entre le local et le global ", dans ZONGO, B. (dir), L'écho de ma langue (enjeux sociaux et culturels de la diversité des langues), TamTam59/Confluence, 2007, pp. 31-37 (en ligne). Disponible sur https:// halshs.archives-ouvertes.fr/halshs-00608595. Consulté le 08. 05.2018.

BULOT T., « Une sociolinguistique prioritaire. Prolégomènes à un développement durable urbain et linguistique ». in Agir ET penser - Les Rencontres de Bellepierre, La Réunion, mai 2008, 7 pages (en ligne en au format pdf). Disponible sur http://www.lrdb.fr. Consulté le 15.03.2018.

BULOT T., «Les étrangers et leur langue à Rennes Métropole : d'une sociolinguistique urbaine à une sociolinguistique prioritaire ", dans BULOT, T. et al (dirs), Intervenir : appliquer, s'impliquer? Paris, L'Harmattan, 2009a, pp. 115-124.

BULOT T., «Introduction. Durabilité et sociolinguistique urbaine. Premiers éléments de discussion ", dans BULOT, T. et al. (dirs), Formes et normes sociolinguistiques (ségrégations et discriminations urbaines), Paris, L'Harmattan, 2009b, pp. 7-13.

BULOT T., « Normes et discrimination(s). Frontières, espaces et langues », Cahiers Internationaux de Sociolinguistique, $\mathrm{n}^{\circ}$ 4, Paris, L'Harmattan, 2013, pp 5-11.

BULOT T. et BAUVOIS C., « Présentation générale. La sociolinguistique urbaine : une sociolinguistique de crise ? Premières considérations ", dans BULOT, T, (dir), Lieux de ville et identité (perspectives en sociolinguistique urbaine), Paris, L'Harmattan, 2004, pp. 7-16.

BULOT T. et VESCHAMBRE V., Mots, traces et marques (Dimensions spatiale et linguistique de la mémoire urbaine, Paris, L'Harmattan, 2006.

DIALLO S., « Cameroun : la musique urbaine, une bonne sauce », article mis en ligne en 2017. Disponible sur https://happyinafrica.com/culture-fr/cameroun-musique-urbaine-bonne-sauce. Consulté le 08. 05.2018.

EYEBE MPESSE J.P., » Au-delà de l'interdiction de la chanson « coller la petite » », article mis en ligne en 2015. Disponible sur http://www.cameroun24.net/ actualitecamerounOpinion_-3AAu_del_C3_A0_de_1_E2_80_99interdiction_de_la_chanso-3-3-25888.html. Consulté le 21.05.2018. 
GODONOU A., « Perversion ; obscénités verbales et dansantes : Lettre Ouverte aux artistes, chanteurs et musiciens camerounais ", article mis en ligne en 2014. Disponible sur https://www. 237online.com/article-49076-cameroun--perversion-obscenites-verbales-et-dansantes-lettreouverte-aux-artistes-chanteurs-musiciens-camerounais.html. Consulté le 21.05.2018.

MEYRAN R., «Les musiques urbaines, ou la subversion des codes esthétiques occidentaux », in EspacesTemps.net. Travaux mis en ligne le 27.01.2014. Disponible sur https:// www.espacestemps.net/articles/les-musiques-urbaines-ou-la-subversion-des-codes-esthetiquesoccidentaux/. Consulté le 16.05.2018.

MORGANE G., « » Coller la petite » : la chanson est censurée au Cameroun ! », article mis en ligne en 2015. Disponible sur http://fr.trace.tv/musique/coller-la-petite-la-chanson-est-censuree-aucameroun/. Consulté le 19.05.2018.

NDOUNGMO E., Le discours de l'obscénité dans la musique urbaine camerounaise contemporaine : essai d'analyse pragmatique, Mémoire de Master, Université de Dschang, (2017).

TNK, «Les musiques urbaines : culture populaire et expression des qualités humaines et attachement à la culture bantou », article mis en ligne en 2016. Disponible sur https:// bantouurbangroove.wordpress.com/2016/04/18/les-musiques-urbaines-culture-populaire-etexpression-des-qualites-humaines-et-attachement-a-la-culture-bantou/. Consulté le 16.05.2018.

\section{NOTES}

1. Dénonciation de la misère ambiante, des injustices sociales et politiques au profit des valeurs morales telles que l'amour, la paix, la justice, le savoir être ou le savoir vivre, etc.

2. Le terme est ici à comprendre comme ce qui relève du quotidien et de la banalité, bref, du relâché qui subvertit volontairement une ou des normes existantes.

3. «(In)justices spatiales, langue(s) et discours : Théorisations politiques et interventions »

4. «Le discours de l'obscénité dans la musique urbaine camerounaise contemporaine: essai d'analyse pragmatique ", Mémoire de Master, Université de Dschang

5. Thèse de doctorat en cours de finalisation sur la chanson rap au Cameroun, Université de Dschang

6. Recherche en cours sur la musique pornographique camerounaise contemporaine et notamment le genre Bikutsi.

7. «La musique camerounaise », in https://fr.wikipedia.org/wiki/Musique_camerounaise. Consulté le 16.05.2018.

8. Il s'agit d'une région autour de Douala dans le Littoral du Cameroun où les filles ont la réputation d'aimer et de savoir faire l'amour.

9. Cette expression en camfranglais est polysémique et peut désigner en fonction du contexte plusieurs choses à la fois. Dans le cas d'espèce il s'agit du sexe, et par extension, de l'amour

10. Dans la région de l'Est-Cameroun avec la même réputation que le cas évoqué plus haut

11. Se dit en français camerounais de ce qui se donne et se prend facilement ou gratuitement

12. Dans la région de l'Ouest-Cameroun réputée pour avoir un taux de natalité très élevé

13. Région de l'Est-Cameroun

14. Se dit d'une situation où l'on donne ou reçoit beaucoup d'argent pendant ou après une prestation, comme chez les artistes par exemple. Dans le cas d'espèce, il s'agit de la situation où la femme fait l'amour avec en contrepartie beaucoup d'argent, de la prostitution en somme. 


\section{RÉSUMÉS}

La musique urbaine camerounaise contemporaine est devenue progressivement l'un des espaces des discours sociaux ou des arts populaires en général où la discrimination positivée du corps a connu un véritable délitement ces dix dernières années. Elle a bousculé les normes (esthétiques, sociales et voire artistiques) avec une évolution thématique au niveau du « bas ventre » qui prône l'obscène et une désacralisation de la nudité et de l'intimité qui étaient jusque-là le socle des valeurs traditionnelles ou culturelles. L'objectif est ici d'explorer le discours artistique comme étant une nouvelle forme de (ré)appropriation et de réinvention de l'espace social public pouvant nécessiter une régulation sociale et politique dans le but de consolider le « vivre ensemble ».

The Cameroon contemporary music has progressiveley become one of the social discourses or popular arts in general where the body negative discrimination has increased these last ten years. It has pushed out the norms (aesthetic, social or artistic) with a thematic evolution at the « under stomach » level which encourages obscenity and a desacralization of the nakeness which was until then the basis of the traditional and cultural values. The main objectif of this article is to explore the artistic discourse as a new form of (re)appropriation and reinvention the public social space which needs a social or a political regulation in term of « living together ».

INDEX

Mots-clés : musique, discriminations, corps, intervention, sexe

Keywords : music, discriminations, body, intervention, sex

\section{AUTEUR}

\section{JEAN-BENOÎT TSOFACK}

Université de Dschang, Cameroun 